\title{
Synthesis of porous silica hollow spheres using sacrificial template for drug delivery applications
}

\author{
Rasoul Safdari $^{\mathrm{a}}$, Shahrara Afshar ${ }^{\mathrm{a}^{*}}$ and Alireza Khosravi ${ }^{\mathrm{b}}$
}

${ }^{a}$ Department of Chemistry, Iran University of Science and Technology, Tehran 16846-13114, Iran

${ }^{b}$ Mycology Research Center, Faculty of Veterinary Medicine, University of Tehran, Tehran 14155-6453, Iran

\section{H R O N I C L E}

Article history:

Received January 22, 2014

Received in revised form

February 02, 2014

Accepted 8 May 2014

Available online

30 May 2014

Keywords:

$\mathrm{SiO}_{2}$ hallow sphere

Drug delivery

Sacrificial template \begin{abstract}
A B S T R A C T
In this work, we report on the synthesis of $\mathrm{SiO}_{2}$ hollow spheres using carbon nanospheres as the sacrificial template by hydrothermal method. The synthesized substrates are in a spherical morphology and uniform size distribution. The effects of hydrothermal process, concentration and the reaction temperature were optimized during synthesis of carbon nanospheres. Infrared spectroscopy (IR), and scanning electronic microscopy (SEM) methods were used for identification of the synthesized products. The synthesized $\mathrm{SiO}_{2}$ nanospheres were used as drug carrier to investigate in vitro release behavior of monoterpenic phenol isomers, carvacrol and thymol, in simulated body fluid (SBF). Ultraviolet-visible spectroscopy (UV-vis) method was carried out to determine the amount of the drugs entrapped in the carrier. The results indicated that $\mathrm{SiO}_{2}$ nanospheres have high ability to adsorb the drugs and there is no need for adjusting the $\mathrm{pH}$ during the adsorption process. The drug release profile shows a three stages pattern and indicates a delayed release action.
\end{abstract}

\section{Introduction}

Morphology of nanostructures plays an important role in determining their properties. Because of low density, high surface area, loading ability, encapsulation of guest molecules, high porosity, ease of production, and suitable biodegradability, hollow nanospheres are of great interest ${ }^{1,2}$. Performance of $\mathrm{SiO}_{2}$ nanospheres is strongly influenced by the size of their porosity and their surface area. $\mathrm{SiO}_{2}$ nanospheres have many applications in medical sciences ${ }^{3}$, biotechnology photovoltaic chromatography ${ }^{4,5}$ and targeted drug delivery ${ }^{6}$. So far, many physical and chemical methods were used to synthesis the $\mathrm{SiO}_{2}$ nanospheres, such as hydrolysis ${ }^{7}$, hydrothermal, sol gel, polymerization ${ }^{8}$, using sacrificial templates ${ }^{9}$, and etc. Among these, sacrificial templates method has attracted much attentions due to its ability to control particle size ${ }^{10}$. By controlling template size and concentration, reaction time and temperature, desired morphology and size of $\mathrm{SiO}_{2}$ nanospheres could be obtained ${ }^{11}$. In this research, $\mathrm{SiO}_{2}$ nanospheres with uniform morphology and size distribution were synthesized

\footnotetext{
* Corresponding author.

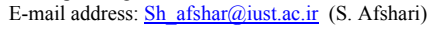


using carbon sacrificial templates. In addition, the effects of temperature, concentration and reaction time were optimized. To create porosity in $\mathrm{SiO}_{2}$ nanospheres, cetyltrimethylammonium bromide (CTAB) was used as surfactant. Exploring of drug loading on porous $\mathrm{SiO}_{2}$ nanospheres showed that these particles have good potential to be a drug carrier with no need to $\mathrm{pH}$ adjustment.

\section{Experimental}

\subsection{Materials and Methods}

All the reagents were of analytical grade and were from Merck Company. Reagents were used without any further purification.

The FT-IR spectra were recorded using $\mathrm{KBr}$ disks on an FT-IR SHIMADZU 8400S infrared spectrometer and absorptions were reported as wave numbers $\left(\mathrm{cm}^{-1}\right)$. Ultraviolet-visible (UVVis) absorption spectrum was measured on a T80 spectrophotometer. The morphologies of particles were observed by scanning electron microscopy (SEM, LEO-1455 VP).

$50 \mathrm{~mL}$ of glucose solution $(0.4 \mathrm{M})$ was transferred into an autoclave receptacle and maintained at $180{ }^{\circ} \mathrm{C}$ for $12 \mathrm{~h}$. The product was dispersed in water using ultrasonic bath, then centrifuged for 20 min at a speed of $6000 \mathrm{rpm}$, and finally washed with ethanol. The procedure of dispersing, centrifuging and washing repeated six more times. After solvent evaporation, carbon nanospheres were obtained.

To synthesize the $\mathrm{SiO}_{2}$ hollow spheres, $0.06 \mathrm{~g}$ carbon nanospheres were dispersed in $100 \mathrm{~mL}$ ethanol, and $0.05 \mathrm{~g}$ CTAB was added to the mixture. The $\mathrm{pH}$ was adjusted at 10.5 by addition of 10 $\mathrm{mL}$ ammonia solution (25\%). Then, while using an ultrasonic bath, $2 \mathrm{~mL}$ of tetraethylorthosilicate (TEOS) was added dropwise to the suspension. After $1 \mathrm{~h}$ sonication, the product was centrifuged and washed with water and ethanol until pH 7 and let to dry. The obtained precipitate $\left(\mathrm{C} @ \mathrm{SiO}_{2}\right)$ was calcined at $600{ }^{\circ} \mathrm{C}$ for $3 \mathrm{~h}$ to burn the carbon cores and obtain the $\mathrm{SiO}_{2}$ hollow nanospheres as final product.

The loading of the monoterpenic phenol isomers (carvacrol and thymol) was carried out by the soaking of $0.01 \mathrm{~g}$ of $\mathrm{SiO}_{2}$ hollow spheres in $5 \mathrm{ml}$ ethanolic solution of drugs $(6.5 \mathrm{mM})$. The suspension was stirred for $48 \mathrm{~h}$. After centrifugation of drug-loaded $\mathrm{SiO}_{2}$ nanospheres, the amount of the drug loaded was measured using the absorption intensity of the drug remained in solution at 420 $\mathrm{nm}$ by means of UV-vis spectroscopy method. In addition, the in vitro release of the drug was performed by soaking the drug-loaded powder in simulated body fluid (SBF), with continuous stirring at $37^{\circ} \mathrm{C}$ in a water bath. The release ration of the drug from the carrier into the solution was measured by examining the concentration of the drug in SBF at different time intervals, by means of UV-vis spectroscopy.

\section{Results and discussion}

IR spectrum of carbon nanospheres shows that they contain carboxyl, ester and alcoholic functional groups on their surface. According to IR spectrum, the peaks at $875-750 \mathrm{~cm}^{-1}, 1460-1000$ $\mathrm{cm}^{-1}, 1715-1700 \mathrm{~cm}^{-1}, 3000-2815 \mathrm{~cm}^{-1}$ and $3700-3000 \mathrm{~cm}^{-1}$ region are attributed to the out of plane aromatic $\mathrm{C}-\mathrm{H}, \mathrm{C}-\mathrm{O}$ stretching, ester stretching, aliphatic $\mathrm{C}-\mathrm{H}$ stretching, and carboxylic acid stretching vibrations, respectively. Comparing Fig. 1, a and b shows that with increasing the hydrothermal time (from 8 to $16 \mathrm{~h}$ ), glucose loses more water and finally polymerizes. Therefore by reducing the oxygen to carbon ratio, the intensity of the $3700-3000 \mathrm{~cm}^{-1}$ peaks are reduced ${ }^{13}$. As shown in IR spectra of glucose core and $\mathrm{SiO}_{2}$ shell before and after calcination (Fig. 2), the functional groups attached to the carbon nanospheres are eliminated that could be due to the complete burning of carbon template. The peaks at 470 and $1110 \mathrm{~cm}^{-1}$ region are attributed to the Si-O bending and stretching vibrations, respectively. The peak around $3500 \mathrm{~cm}^{-1}$ could be due to the $\mathrm{O}-\mathrm{H}$ stretching vibration of water. 


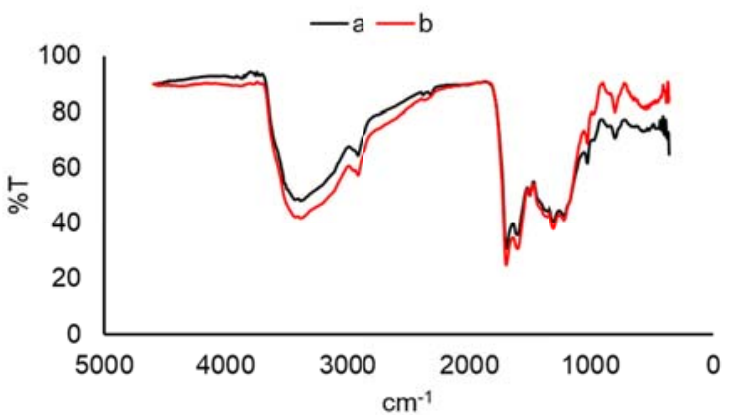

Fig. 1. IR Spectra of the glucose core (a) after 16h and (b) after $8 \mathrm{~h}$ heating in a hydrothermal system

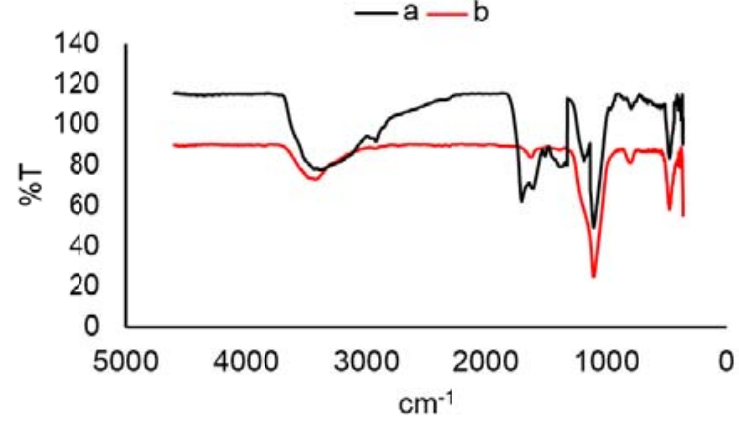

Fig 2. IR Spectra of $\mathrm{C} @ \mathrm{SiO}_{2}$ before (a) and after calcination (b) at $600 \circ \mathrm{C}$ for $3 \mathrm{~h}$

The SEM images (Fig. 3) confirm the morphology of carbon nanospheres. The SEM images show, the size of the carbon nanospheres at different hydrothermal reaction time (Fig 3 a $-d$ ). According to Fig. 3, to obtain the carbon nanospheres with the desired uniform morphology and size, the optimum time for hydrothermal process is $12 \mathrm{~h}$. Longer hydrothermal process is undesirable, because it causes an agglomeration of nanospheres ${ }^{14}$.
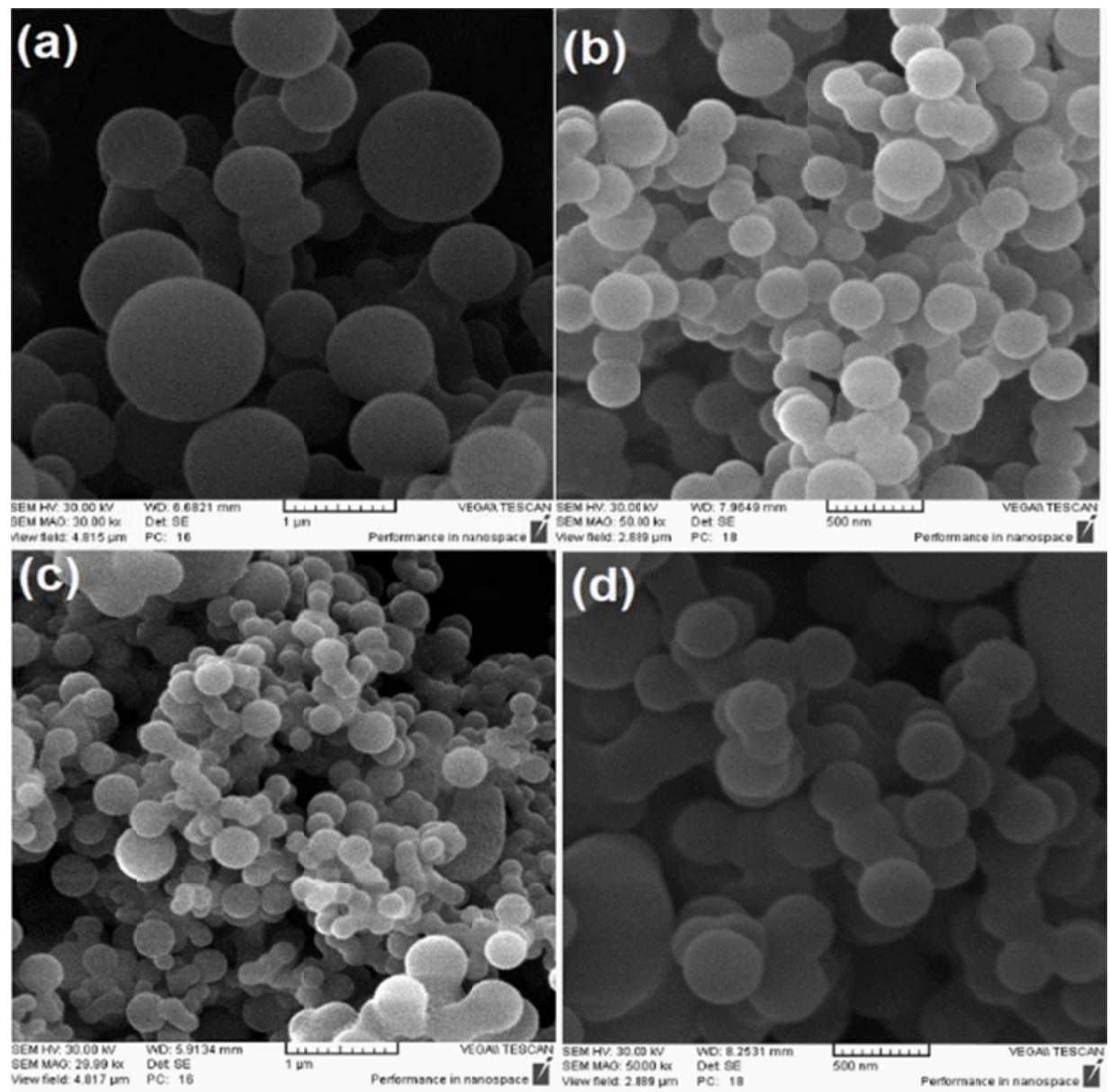

Fig 3. SEM images of glucose spheres after (a) 8, (b) 12, (c) 14 and (d) 16 h heating in a hydrothermal system 

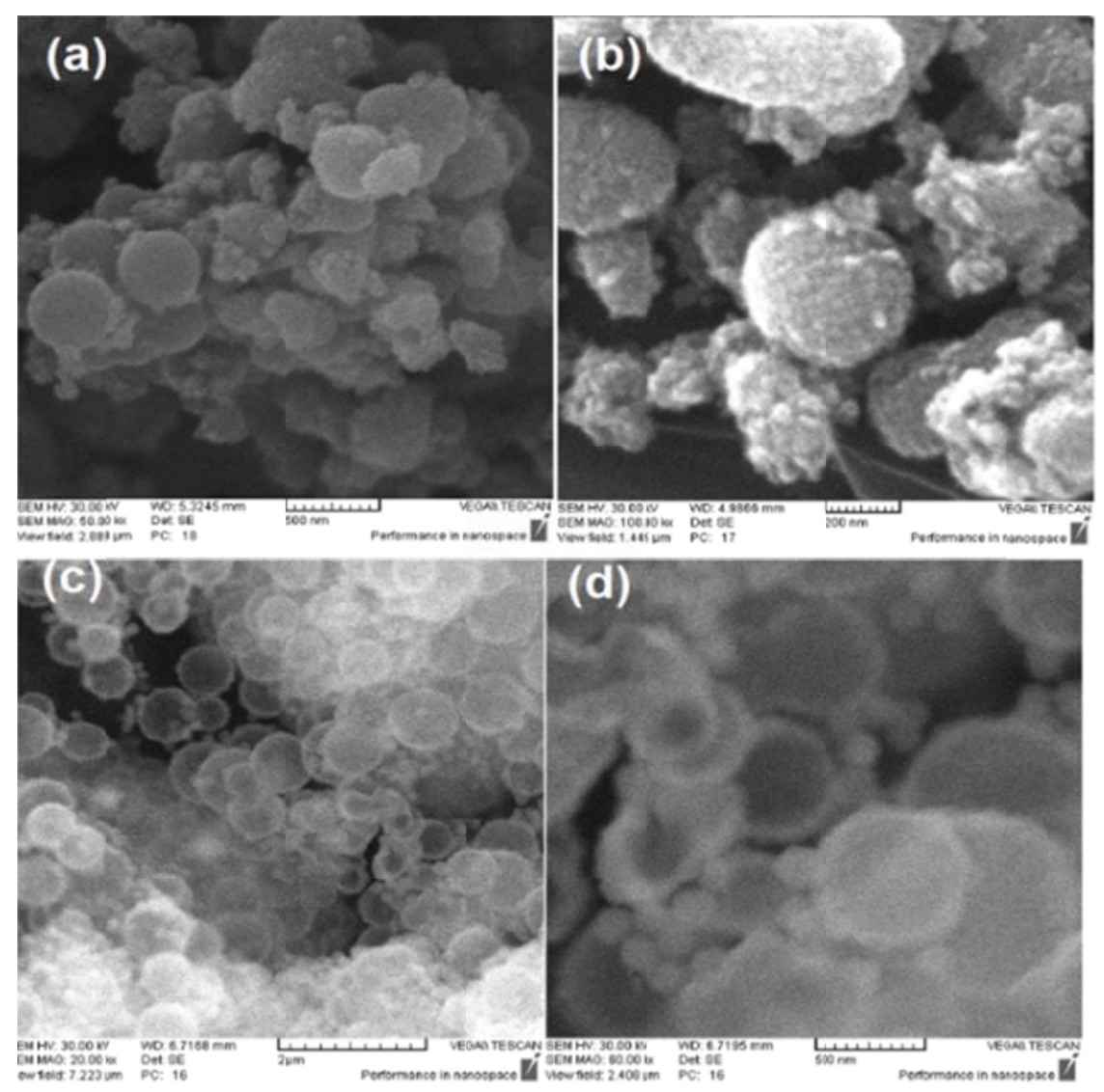

Fig 4. SEM images of $\mathrm{C} @ \mathrm{SiO}_{2}$ shell before calcination $(\mathrm{a}, \mathrm{b})$ and $\mathrm{SiO}_{2}$ hallow shells remained after calcination of $\mathrm{C} @ \mathrm{SiO}_{2}(\mathrm{c}, \mathrm{d})$

The SEM images of $\mathrm{C} @ \mathrm{SiO}_{2}$ nanospheres before calcination are shown in Fig $4(\mathrm{a}, \mathrm{b})$. The $\mathrm{SiO}_{2}$ particles around the $\mathrm{C}$ cores are clearly obvious. SEM images in Fig 4 (c,d) show the hollow silica shells and it is obvious that after calcination, carbonaceous materials were burned out completely and $\mathrm{SiO}_{2}$ hollow spheres were remained. Results of SEM images are in good agreement with IR data.

To explore the amount of drug loading on the porous $\mathrm{SiO}_{2}$ nanospheres, UV/Vis spectrophotometry was used. Fig. 5 shows the UV/Vis spectra of the ethanolic solution of the drugs $(6.5 \mathrm{mM})$ in presence of $\mathrm{SiO}_{2}$ hollow spheres after $6,12,24$ and $48 \mathrm{~h}$. As it is obvious due to the porosity of $\mathrm{SiO}_{2}$ nanospheres, the drug molecules have adsorbed completely after $48 \mathrm{~h}$. $\mathrm{The}^{\mathrm{SiO}} 2^{-}$ drug conjugation methods include silanization and electrostatic attractions. The monoterpenic phenol isomers (carvacrol and thymol) contain hydroxyl groups, which can interact with silanol groups in silica via hydrogen bonding.

The drug release in vitro has been investigated also by means of UV-Vis spectroscopy. The spectra of a suspension of the $\mathrm{SiO}_{2}$ loaded with drugs have been taken at the beginning of making the suspension, after $30 \mathrm{~min}, 1,2,5 \mathrm{~h}$ and then every $3 \mathrm{~h}$ for a total of $26 \mathrm{~h}$. Fig. 6 shows the release profile of the drugs from the hollow carrier. As can be seen, by the passage of time, the drug release $\%$ in solution increases which is due to the increment of the drugs concentration in the simulated body fluid (SBF). The drug release has happened in three stages. At the first stage, about $43 \%$ of the drug has been discharged within $125 \mathrm{~min}$, which is probably due to the rapid release of the drug loosely adsorbed on the surface of the nanoshells or free drug molecules. However, this type of fast release is not probably favorable for the practical controlled release of drugs. The second stage has lasted to about $650 \mathrm{~min}$ ( $11 \mathrm{~h}$ ) in which $37 \%$ of the drug released from the nanoshells and in the third stage, which has lasted up to $26 \mathrm{~h}, 8 \%$ of the drug released. This last stage could be related to the release of the drug trap inside the hallow spheres of the carrier that causes a slow releasing to the target cells ${ }^{12}$. In addition, it is probable that slow release is pertained to the strong chemical 
adsorption of some drug molecules in the silica pores. Lack of too many functional groups in carvacrol and thymol for interaction with $\mathrm{SiO}_{2}$ nanospheres, results in encapsulation that could be very important for their slow releasing ${ }^{10}$. So counting on the last two stages, it can be concluded that the porous hollow silica nanospheres, as drug carrier, markedly delayed the release of the carvacrol and thymol drugs and can be used in drug delivery applications.

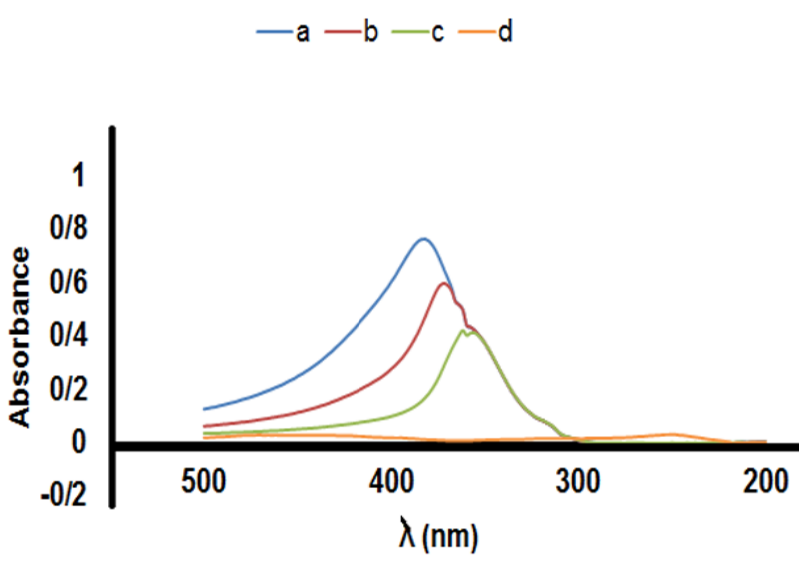

Fig. 5. UV/Vis spectra of the ethanolic solution of the drugs $(6.5 \mathrm{mM})$ in presence of $\mathrm{SiO}_{2}$ hollow spheres after (a) 6, (b)12, (c) 24 and (d) $48 \mathrm{~h}$

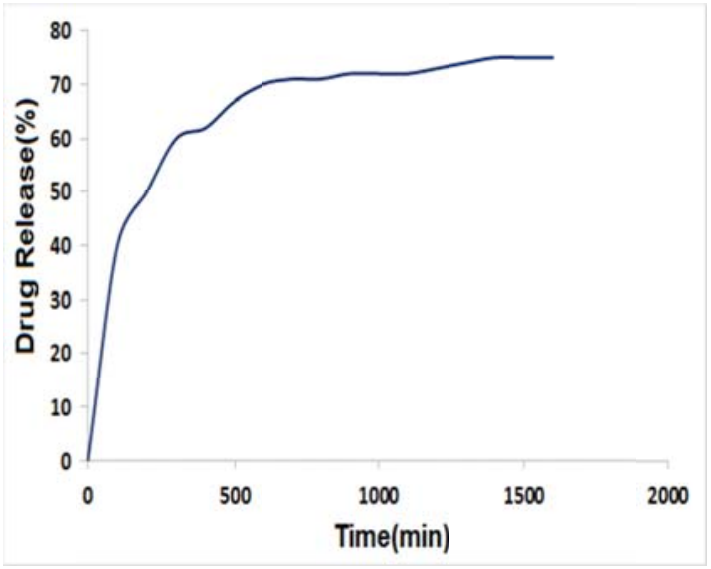

Fig 6. The release profile of the drugs from the hollow carrier into SBF

\section{Conclusion}

In summary, $\mathrm{SiO}_{2}$ hollow spheres were successfully synthesized using carbon nanospheres as sacrificial template by hydrothermal method. The SEM images show that $\mathrm{SiO}_{2}$ hollow spheres with uniform morphology and size distribution were synthesized. Also by controlling the hydrothermal process conditions like concentration and the reaction time and temperature, a tunable controlled size of $\mathrm{SiO}_{2}$ can be prepared using carbon sacrificial templates, which have potential applications in drug delivery. The $\mathrm{SiO}_{2}$ nanospheres have high ability for adsorbing drug and there is no need to adjust $\mathrm{pH}$ during adsorption. The results have also shown that $\mathrm{SiO}_{2}$ nanospheres can release drugs at different rates.

\section{References}

1 Kim J. H., Son M., Sohn Y., and Shin W. G. (2013) Hollow $\mathrm{SiO}_{2}$ nanospheres: One-step synthesis by introducing guest ag nanoparticles and an irradiating electron beam under ambient condition. Aerosol. Air Qual. Res., 13(1), 415-420.

2 Zou H., Wu S., and Shen J. (2008) Polymer/silica nanocomposites: Preparation, characterization, properties, and applications. Chem. Rev, 108(9), 3893-3957.

3 Liu D., Mäkilä E., Zhang H., Herranz B., Kaasalainen M., Kinnari P., Salonen J., Hirvonen J., and Santos H. A. (2013) Nanostructured porous silicon-solid lipid nanocomposite: Towards enhanced cytocompatibility and stability, reduced cellular association, and prolonged drug release. Adv. Funct. Mater., 23(15), 1893-1902.

4 Zheng-Wei M., Ling H., Qing-He Z., and Chang-You G. (2012) Fabrication of porous silica microspheres under the assistance of solid template. Chem. Res. Chin.Univ., 28(3), 546-549.

5 Li Z., Zhu L., Liu Q., Du Y., and Wang F. (2013) A facile approach to synthesize $\mathrm{SiO}_{2} \cdot \mathrm{re}_{2} \mathrm{O}_{3}$ $(\mathrm{re}=\mathrm{Y}, \mathrm{Eu}, \mathrm{La}, \mathrm{Sm}, \mathrm{Tb}, \mathrm{Pr})$ hollow sphere and its application in drug release. Nanoscale Res. Lett., 8(1), 1-10.

6 Salonen J., Kaukonen A. M., Hirvonen J., and Lehto V. P. (2008) Mesoporous silicon in drug delivery applications. J. Pharm. Sci., 97(2), 632-653. 
7 Liu H., Li H., Ding Z., Fu A., Guo P., Yu J., Wang C., and Zhao X. S. (2012) Preparation of porous hollow $\mathrm{SiO}_{2}$ spheres by a modified stöber process using mf microspheres as templates. $J$. Cluster Sci., 23(2), 273-285.

8 Stöber W., Fink A., and Bohn E. (1968) Controlled growth of monodisperse silica spheres in the micron size range. J. Colloid Interface Sci., 26(1), 62-69.

9 Das D., Parida K., and Mishra B. (2009) Synthesis and surface properties of silica spheres with core shell structure by one convenient method. Adv. Mater. Sci. Eng., 2009.

10 Deng T.-S., and Marlow F. (2012) Synthesis of monodisperse polystyrene@ vinyl-SiO $\mathrm{S}_{2}$ coreshell particles and hollow $\mathrm{SiO}_{2}$ spheres. Chem. Mater., 24(3), 536-542.

11 Wang Z.-B., Li C.-Z., Gu D.-M., and Yin G.-P. (2013) Carbon riveted ptru/c catalyst from glucose in-situ carbonization through hydrothermal method for direct methanol fuel cell. $J$. Power Sources, 238(283-289.

12 Gultepe E., Nagesha D., Sridhar S., and Amiji M. (2010) Nanoporous inorganic membranes or coatings for sustained drug delivery in implantable devices. Adv. Drug Delivery Rev., 62(3), 305315.

13 Li B., Yang X., Xia L., Majeed M. I., and Tan B. (2013) Hollow microporous organic capsules. Sci. Rep., 3.

14 Sevilla M., and Fuertes A. B. (2009) The production of carbon materials by hydrothermal carbonization of cellulose. Carbon, 47(9), 2281-2289. 